\title{
Article
}

\section{Inversion improves the recognition of facial expression in thatcherized images}

\author{
Psalta, Lilia and Andrews., Timothy J \\ Available at http://clok.uclan.ac.uk/13504/ \\ Psalta, Lilia and Andrews., Timothy J (2014) Inversion improves the \\ recognition of facial expression in thatcherized images. Perception, 43 (8). pp. \\ 715-730. ISSN 0301-0066
}

It is advisable to refer to the publisher's version if you intend to cite from the work. http://dx.doi.org/10.1068/p7755

For more information about UCLan's research in this area go to

http://www.uclan.ac.uk/researchgroups/ and search for <name of research Group>.

For information about Research generally at UCLan please go to http://www.uclan.ac.uk/research/

All outputs in CLoK are protected by Intellectual Property Rights law, including Copyright law. Copyright, IPR and Moral Rights for the works on this site are retained by the individual authors and/or other copyright owners. Terms and conditions for use of this material are defined in the policies page.

\section{CLoK}

Central Lancashire online Knowledge www.clok.uclan.ac.uk

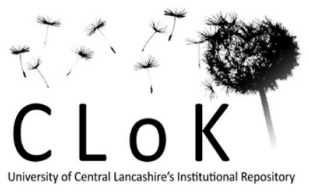




\title{
Inversion improves the recognition of facial expression in thatcherized images
}

\author{
Lilia Psalta, Timothy J Andrews§ \\ Department of Psychology and York Neuroimaging Centre, University of York, York YO10 5DD, UK; \\ e-mail: timothy.andrews@york.ac.uk \\ Received 20 March 2014, in revised form 24 June 2014, published online 19 August 2014
}

\begin{abstract}
The Thatcher illusion provides a compelling example of the face inversion effect. However, the marked effect of inversion in the Thatcher illusion contrasts to other studies that report only a small effect of inversion on the recognition of facial expressions. To address this discrepancy, we compared the effects of inversion and thatcherization on the recognition of facial expressions. We found that inversion of normal faces caused only a small reduction in the recognition of facial expressions. In contrast, local inversion of facial features in upright thatcherized faces resulted in a much larger reduction in the recognition of facial expressions. Paradoxically, inversion of thatcherized faces caused a relative increase in the recognition of facial expressions. Together, these results suggest that different processes explain the effects of inversion on the recognition of facial expressions and on the perception of the Thatcher illusion. The grotesque perception of thatcherized images is based on a more orientation-sensitive representation of the face. In contrast, the recognition of facial expression is dependent on a more orientation-insensitive representation. A similar pattern of results was evident when only the mouth or eye region was visible. These findings demonstrate that a key component of the Thatcher illusion is to be found in orientation-specific encoding of the features of the face.
\end{abstract}

Keywords: face, Thatcher illusion, emotion, expression

\section{Introduction}

The impairment in the perception and recognition of facial identity following inversion is a well-established phenomenon in face perception (Diamond \& Carey, 1986; Valentine, 1988; Yin, 1969). Although the face inversion effect is a robust finding across many aspects of face processing, the effect of inversion on the perception of facial expression is less clear. Inversion has been shown to have a small effect on the recognition of negative emotions, but little effect on the recognition of positive emotions, such as happiness (Calvo \& Nummenmaa, 2008; Fallshore \& Bartholow, 2003; Goren \& Wilson, 2006; McKelvie, 1995; Prkachin, 2003).

The relatively small and inconsistent cost of inversion on the perception of facial expression contrasts to the substantial effect of inversion found in the Thatcher illusion. Turning the eyes and the mouth upside-down relative to the rest of the face (a transform we will call 'thatcherization') results in the perception of a grotesque facial expression when the face is upright, but when the image is inverted the grotesque appearance is no longer visible (Thompson, 1980).

The effect of inversion on the perception of facial expression seen in the Thatcher illusion is widely attributed to disruption of configural processing. The distinction between the processing of local features (such as eyes and mouths) and configural properties based on spatial interrelationships between the features of the face was introduced by Carey and Diamond (1977), who maintained that configural processing is impaired by inversion whereas feature processing is largely equivalent across upright and inverted faces. For upright faces, they argued that both configural and featural processing are possible, 
whereas for inverted faces only feature processing can be used. From this perspective, it follows that the cause of the disruptive effect of inversion in the Thatcher illusion reflects a disruption of configural processing, and many researchers have adopted this intuitively appealing line of reasoning.

The aim of this study was to address the discrepancy between the effect of inversion on the recognition of facial expressions and the perception of the Thatcher illusion. First, we compared the relative effect of inversion and thatcherization on the recognition of expression from a validated set of face stimuli (Young, Perrett, Calder, Sprengelmeyer, \& Ekman, 2002). Our prediction was that the disruption to the canonical presentation of facial features in the thatcherized images should have a large effect on the recognition of expression. In contrast, inversion of face images should have a much smaller effect on the recognition of expression. Next, we asked how inversion affects the recognition of facial expression in thatcherized faces. On the basis of previous studies, our prediction was that the inversion of thatcherized images should lead to a further modest reduction in the recognition of facial expression. However, in a thatcherized face the features have an orientation typically found in upright faces. So, it is also possible that recognition performance will be improved. Previous studies have suggested that the Thatcher illusion can be explained by the absence of configural processing in inverted faces (Bartlett \& Searcy, 1993; Carey \& Diamond, 1977). To address the importance of configural processing, we asked whether a similar pattern of results is evident when the key expressive features (mouth region or eye region) are shown in isolation. If the same pattern of results can be found when only featural information is present, this would challenge configural explanations of the Thatcher illusion. On the other hand, if the effects seen in the whole face are dependent on configural processing, we would not expect to see a similar pattern of results when only the eye or mouth regions are visible.

\section{Materials and methods}

\subsection{Participants}

Twelve participants took part in the experiment 1 (six female; mean age $=20.5$ years, \pm 1.8 years), and twenty participants took part in experiment 2 (sixteen female; mean age $=19.1$ years, \pm 1.6 years). The study was approved by the Psychology Department Ethics Committee at the University of York. Participants were students from the University of York.

\subsection{Stimuli}

Face stimuli were Ekman faces selected from the Facial Expressions of Emotion-Stimuli and Tests (FEEST) set (Young et al., 2002). Six individuals posing different expressions were selected based on the following three main criteria: (i) a high-recognition rate for all expressions [mean recognition rate in a six-alternative forced-choice (6-AFC) experiment: 94\%; Young et al., 2002], (ii) consistency of the action units (muscle groups) across different individuals posing a particular expression, and (iii) visual similarity of the posed expression across individuals. Each face image was thatcherized by inverting the mouth and eyes by $180^{\circ}$. Visual stimuli $(7 \times 11 \mathrm{deg}$ : whole face; $7 \times 2 \mathrm{deg}$ : mouth or eye region $)$ were presented on a computer monitor at a distance of approximately $57 \mathrm{~cm}$ from the participants. NBS Presentation (http://www.neurobs.com) stimulus delivery software was used to present images.

\subsection{Design}

Figure 1a shows examples of images from experiment 1 . We compared the effect of inversion and thatcherization on the recognition of facial expressions of emotion using whole face images. There were 6 facial expressions: neutral, happiness, anger, disgust, fear, and sadness. Images were presented in an upright or inverted orientation. They could also be normal or thatcherized. In total, there were 24 conditions that included 6 expressions $\times 4$ conditions (upright normal, inverted normal, upright thatcherized, inverted thatcherized). 
Individual images from each condition were presented for $800 \mathrm{~ms}$ followed by a $2 \mathrm{~s}$ interstimulus fixation screen. Participants were instructed to press a button to indicate which expression they had seen (6-AFC). There were 24 trials for each condition and a total of 576 trials for the whole experiment. Trials were presented in a counterbalanced, pseudorandomized order.

Figures $1 \mathrm{~b}$ and $1 \mathrm{c}$ show examples of images from experiment 2 . The design and procedure for experiment 2 was identical to experiment 1 . The only difference was that participants judged facial expressions of emotion from face images in which either only the mouth region or only the eye region was visible.


(b)
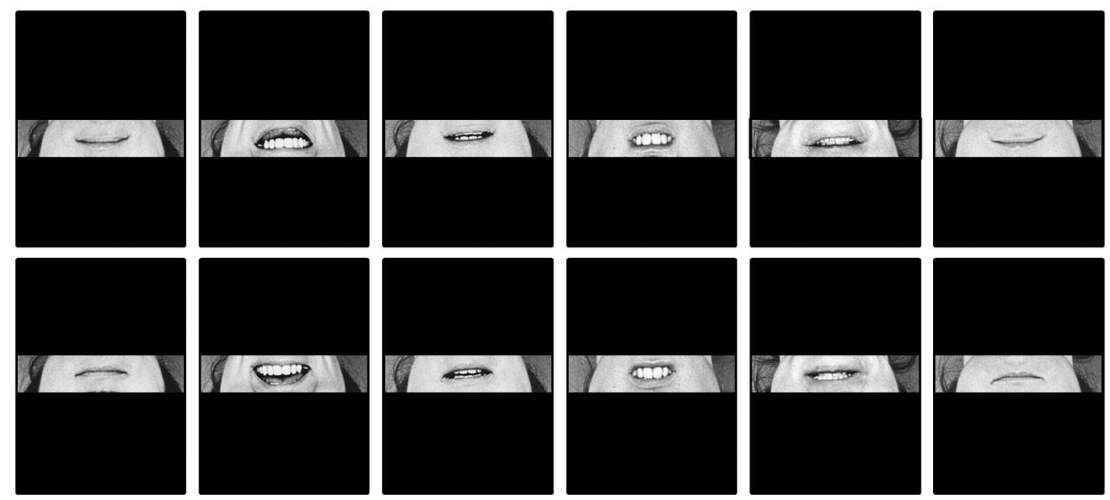

(c)

Figure 1. Example images from experiment 1 and experiment 2. (a) Whole face images showing the 6 facial expressions of emotion: neutral, happy, fear, disgust, anger, sad (left to right). (b, c) The corresponding images from the eye region and mouth region, respectively. Images were shown in a normal (top row) or thatcherized configuration (bottom row). Invert page for the upright view of images. 


\section{Results}

\subsection{Experiment 1}

3.1.1 Recognition accuracy (whole face). The aim of experiment 1 was to determine the effect of inversion on the recognition of expression in normal and thatcherized whole faces. Figure 2 shows the recognition accuracy for each facial expression. A three-way ANOVA with expression (neutral, happy, fear, disgust, anger, sad), condition (normal, thatcherized), and orientation (upright, inverted) was performed on the data. This revealed a significant effect of expression $\left(F_{5,55}=16.8, p<0.001\right)$ and condition $\left(F_{1,11}=53.0, p<0.001\right)$. There was a nonsignificant trend for an effect of orientation $\left(F_{1,11}=4.5, p=0.06\right)$, but there was a significant interaction between expression $\times$ orientation $\left(F_{5,55}=9.9, p<0.001\right)$. This suggests that inversion had a different effect on different emotional expressions. Finally, there was a significant interaction between condition $\times$ orientation $\left(F_{1,11}=44.3, p<0.001\right)$. This suggests that inversion had a different effect on the recognition of expression in normal compared with thatcherized images. To determine how the perception of different facial expressions is affected by orientation and thatcherization, a 2 (condition) $\times 2$ (orientation) ANOVA was performed independently for each expression.
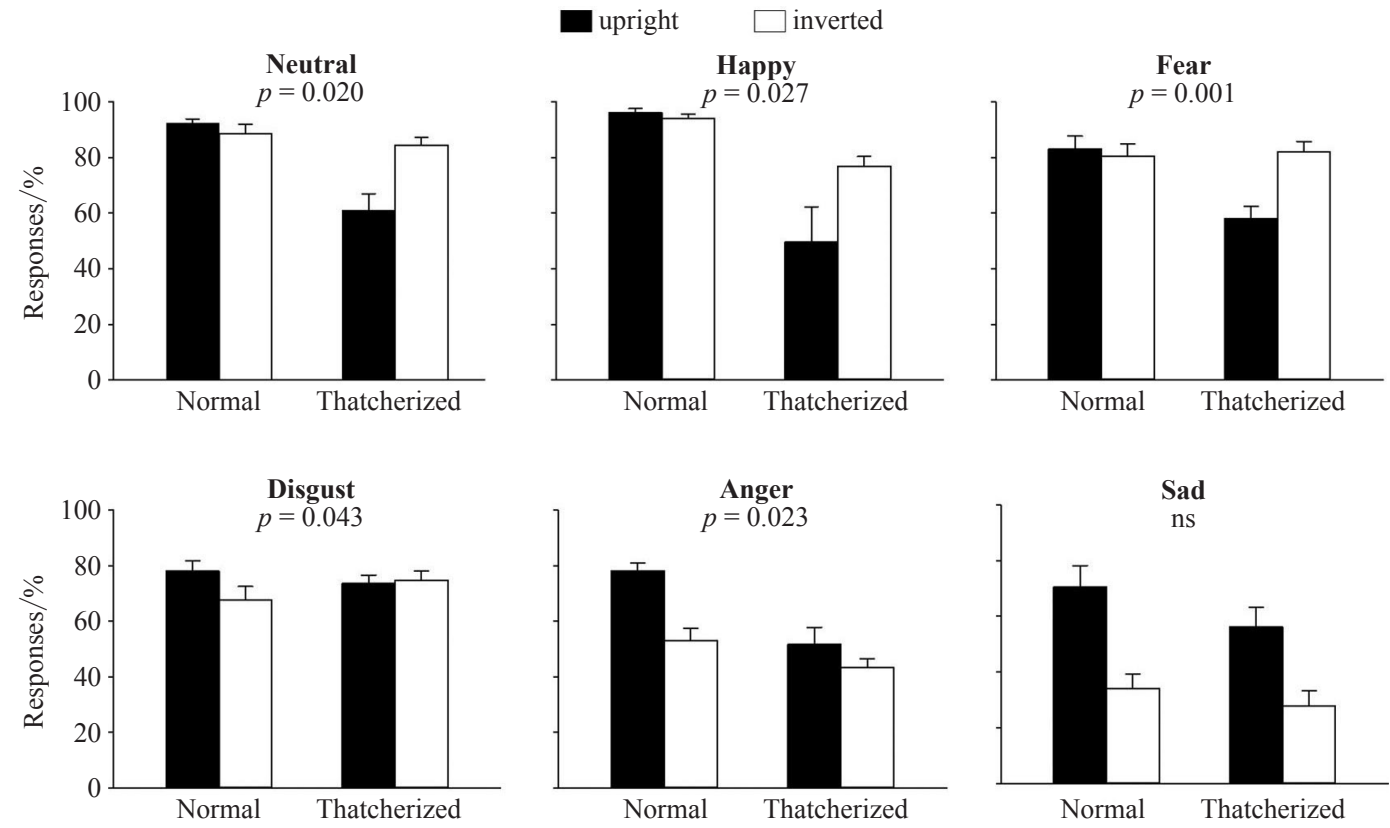

Figure 2. Recognition of facial expression in the whole face. Normal and thatcherized images were presented in an upright or inverted orientation. There was a significant interaction between the orientation of the image and whether the image was normal or thatcherized. This effect was due to lower recognition of inverted compared with upright normal faces, but higher recognition of inverted compared with upright thatcherized faces. The $p$-values for the interaction are shown for each emotional expression. Error bars represent \pm standard error across participants.

For neutral, there was a significant effect of condition $\left(F_{1,11}=30.3, p<0.001\right)$. The effect of condition was due to lower recognition of thatcherized $(72.7 \pm 4.4)$ compared with normal $(90.5 \pm 2.5)$ faces. There was also an effect of orientation $\left(F_{1,11}=6.9, p<0.05\right)$ and a significant interaction between condition $\times$ orientation $\left(F_{1,11}=7.3, p<0.05\right)$. This interaction is explained by no difference in recognition between inverted $(88.5 \pm 3.4)$ and upright $(92.4 \pm 1.5)$ normal faces $\left(t_{11}=0.15, p=0.89\right)$, but a higher recognition of inverted $(84.4 \pm 2.8)$ compared with upright $(61.1 \pm 5.9)$ thatcherized faces $\left(t_{11}=3.7, p<0.01\right)$. Finally, the recognition of upright normal faces was significantly higher than the recognition of inverted thatcherized faces $\left(t_{11}=2.7, p<0.05\right)$. 
For happy, there was a significant effect of condition $\left(F_{1,11}=25.8, p<0.001\right)$. The effect of condition was due to lower recognition of thatcherized (63.3 \pm 8.1$)$ compared with normal $(94.0 \pm 4.3)$ faces. There was no significant effect of orientation $\left(F_{1,11}=2.7, p=0.13\right)$, but there was a significant interaction between condition $\times$ orientation $\left(F_{1,11}=6.5, p<0.05\right)$. This interaction is explained by no difference in recognition between inverted $(91.7 \pm 2.9)$ and upright $(96.2 \pm 1.4)$ normal faces $\left(t_{11}=1.6, p=0.14\right)$, but a higher recognition of inverted $(76.9 \pm 3.5)$ compared to upright $(49.7 \pm 12.7)$ thatcherized faces $\left(t_{11}=2.6, p<0.05\right)$. Finally, the recognition of upright normal faces was significantly higher than the recognition of inverted thatcherized faces $\left(t_{11}=6.3, p<0.001\right)$.

For fear, there was a significant effect of condition $\left(F_{1,11}=10.8, p<0.01\right)$. The effect of condition was due to lower recognition of thatcherized $(67.0 \pm 4.0)$ compared with normal $(81.8 \pm 4.5)$ faces. There was also an effect of orientation $\left(F_{1,11}=6.5, p<0.05\right)$ and a significant interaction between condition $\times$ orientation $\left(F_{1,11}=23.2, p<0.01\right)$. This interaction is explained by no difference in recognition between inverted $(80.6 \pm 4.3)$ and upright $(83.0 \pm 4.7)$ normal faces $\left(t_{11}=1.1, p=0.30\right)$, but a higher recognition for inverted $(81.9 \pm 3.9)$ compared with upright $(58.0 \pm 4.4)$ thatcherized faces $\left(t_{11}=4.5, p<0.001\right)$. Finally, the recognition of upright normal faces was not significantly different from the recognition of inverted thatcherized faces $\left(t_{11}=0.9, p=0.37\right)$.

For disgust, there was no significant effect of condition $\left(F_{1,11}=0.0, p=0.96\right)$ and orientation $\left(F_{1,11}=2.8, p=0.12\right)$. However, there was a significant interaction between condition $\times$ orientation $\left(F_{1,11}=5.2, p<0.05\right)$. This interaction is explained by no difference in recognition between inverted $(74.7 \pm 3.5)$ and upright $(73.6 \pm 3.0)$ thatcherized faces $\left(t_{11}=0.4\right.$, $p=0.70)$, but a higher recognition for upright $(78.1 \pm 3.6)$ compared with inverted $(67.7 \pm 4.7)$ normal faces $\left(t_{11}=2.6, p<0.05\right)$. Finally, the recognition of upright normal faces was not significantly different from the recognition of inverted thatcherized faces $\left(t_{11}=1.1, p=0.29\right)$.

For anger, there was a significant effect of condition $\left(F_{1,11}=46.7, p<0.001\right)$. The effect of condition was due to lower recognition of thatcherized (47.6 \pm 4.6$)$ compared with normal $(65.6 \pm 3.6)$ faces. There was also an effect of orientation $\left(F_{1,11}=15.8, p<0.01\right)$ and a significant interaction between condition $\times$ orientation $\left(F_{1,11}=6.9, p<0.05\right)$. This interaction is explained by a lower recognition of inverted $(53.1 \pm 4.2)$ compared with upright $(78.1 \pm 2.8)$ normal faces $\left(t_{11}=5.9, p<0.001\right)$, but no difference in recognition for inverted $(43.4 \pm 3.1)$ compared with upright $(51.7 \pm 6.0)$ thatcherized faces $\left(t_{11}=1.4, p=0.18\right)$. Finally, the recognition of upright normal faces was significantly higher than the recognition of inverted thatcherized faces $\left(t_{11}=10.4, p<0.001\right)$.

For sad, there was a significant effect of condition $\left(F_{1,11}=9.4, p<0.01\right)$. The effect of condition was due to lower recognition of thatcherized $(42.1 \pm 6.3)$ compared with normal $(52.3 \pm 6.5)$ faces. There was also an effect of orientation $\left(F_{1,11}=45.0, p<0.001\right)$. The effect of orientation was due to higher recognition of upright $(63.4 \pm 7.4)$ compared with inverted faces $(30.9 \pm 5.4)$. There was no significant interaction between condition $\times$ orientation $\left(F_{1,11}=2.1, p=0.18\right)$.

To investigate the patterns of errors, a confusion matrix was generated (figure 3 ). This shows how participants responded to different emotional expressions. The majority of responses were evident along the diagonal (correct). It is also interesting to note that the pattern of incorrect responses was not obviously different for normal or thatcherized images. For example, the correlation in correct performance between upright normal and inverted normal was $r=0.94(p<0.001)$. Similarly, the correlation in correct performance between upright normal and upright thatcherized was $r=0.95(p<0.001)$. Finally, the correlation between upright thatcherized and inverted thatcherized was $r=0.91(p<0.001)$. Together, this suggests that the effects of inversion and thatcherization reflect a lower number of correct responses rather than a different pattern of response. 


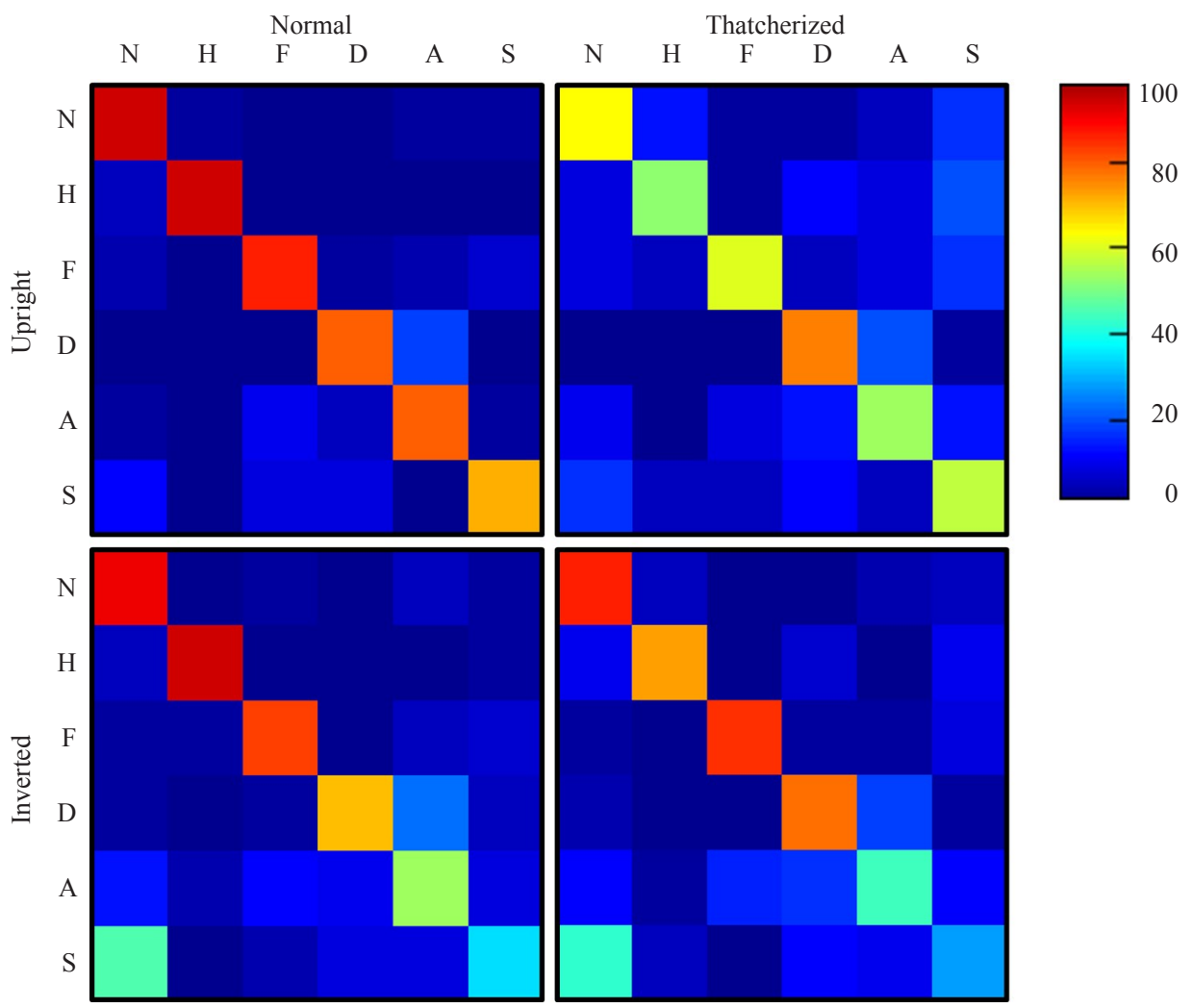

Figure 3. [In colour online, see http://dx.doi.org/10.1068/p7755] Confusion matrices for the presented and perceived emotional expressions in whole faces. The $y$-axis represents the expression that was presented, and the $x$-axis represents the expression that was reported. Correct performance is shown along the diagonal elements, whereas errors or confusion are shown on the off-diagonal elements. The colour bar represents accuracy in \%. Note: $\mathrm{N}=$ neutral; $\mathrm{H}=$ happy; $\mathrm{F}=$ fear; $\mathrm{D}=\operatorname{disgust} ; \mathrm{A}=\operatorname{anger} ; \mathrm{S}=$ sad.

3.1.2 Reaction time (whole face). A three-way ANOVA with expression $\times$ condition $\times$ orientation was performed on the reaction time data in experiment 1 (table 1). There was an effect of orientation $\left(F_{1,7}=6.6, p<0.05\right)$. This was due to lower RT to upright $(1409 \pm 85 \mathrm{~ms})$ compared with inverted $(1598 \pm 99 \mathrm{~ms})$ faces. There was a significant effect of condition $\left(F_{1,7}=16.1\right.$ $p<0.01)$. This was due to lower RT to normal $(1456 \pm 88 \mathrm{~ms})$ compared with thatcherized $(1551 \pm 96 \mathrm{~ms})$ faces. Finally, there was also an effect of expression $\left(F_{5,35}=7.5, p<0.001\right)$. This was due to differences in RT across the different expressions (neutral: $1401 \pm 157 \mathrm{~ms}$; happy: $1401 \pm 160$; fear: $1586 \pm 151 \mathrm{~ms}$; disgust: $1443 \pm 154 \mathrm{~ms}$; anger: $1624 \pm 159 \mathrm{~ms}$; sad: $1568 \pm 175 \mathrm{~ms}$ ). There was a significant interaction between condition $\times$ expression

Table 1. Average reaction time values (ms) for correct responses to emotional expression to the whole face in experiment 1.

\begin{tabular}{llllll}
\hline & \multicolumn{2}{l}{ Normal } & & \multicolumn{2}{l}{ Thatcherized } \\
\cline { 2 - 3 } \cline { 6 - 6 } & upright & inverted & & upright & inverted \\
\hline Neutral & $1257.2 \pm 269.3$ & $1470.6 \pm 374.4$ & & $1401.7 \pm 263.2$ & $1475.0 \pm 346.5$ \\
Happy & $1219.0 \pm 283.4$ & $1427.2 \pm 299.3$ & & $1430.4 \pm 227.4$ & $1527.3 \pm 468.0$ \\
Fear & $1477.8 \pm 305.8$ & $1662.6 \pm 322.8$ & & $1544.9 \pm 302.4$ & $1659.7 \pm 278.9$ \\
Disgust & $1344.6 \pm 333.6$ & $1572.4 \pm 289.2$ & & $1308.8 \pm 299.6$ & $1545.3 \pm 309.0$ \\
Anger & $1430.4 \pm 298.6$ & $1658.0 \pm 315.2$ & & $1642.8 \pm 357.9$ & $1764.5 \pm 299.0$ \\
Sad & $1388.4 \pm 281.7$ & $1568.8 \pm 282.1$ & & $1473.4 \pm 298.9$ & $1842.4 \pm 533.8$ \\
\hline
\end{tabular}


$\left(F_{5,35}=2.6, p<0.05\right)$, but no significant interaction of expression $\times$ orientation $\left(F_{5,35}=0.7\right.$, $p=0.61)$ and no significant interaction between expression $\times$ condition $\times$ orientation $\left(F_{5,35}=0.8, p=0.44\right)$.

\subsection{Experiment 2}

The aim of experiment 2 was to determine the effect of inversion on the recognition of facial expressions in normal and thatcherized faces when only the eye or mouth region was shown.

3.2.1 Recognition accuracy (eye region). Figure 4 shows the percentage-correct recognition for each facial expression in the eye region. To determine the effect of inversion and thatcherization, we performed a three-way ANOVA with expression (neutral, happy, fear, disgust, anger, sad), condition (normal, thatcherized), and orientation (upright, inverted). There was a significant effect of expression $\left(F_{5,95}=55.3, p<0.001\right)$, a significant effect of condition $\left(F_{1,19}=74.8, p<0.001\right)$, and a significant effect of orientation $\left(F_{1,19}=135.6, p<0.001\right)$. There was also a significant condition $\times$ orientation interaction $\left(F_{1,19}=91.9, p<0.001\right)$. This suggests that inversion has a different effect on the recognition of facial expression in normal and thatcherized faces. There was also a significant expression $\times$ condition interaction $\left(F_{5,95}=7.6, p<0.001\right)$ and expression $\times$ orientation interaction $\left(F_{5,95}=26.8, p<0.001\right)$. The interaction between expression $\times$ condition $\times$ orientation was also significant $\left(F_{5,95}=28.9\right.$, $p<0.001)$. To determine how the perception of different facial expressions is affected by orientation and thatcherization, a 2 (condition) $\times 2$ (orientation) ANOVA was performed independently for each expression.
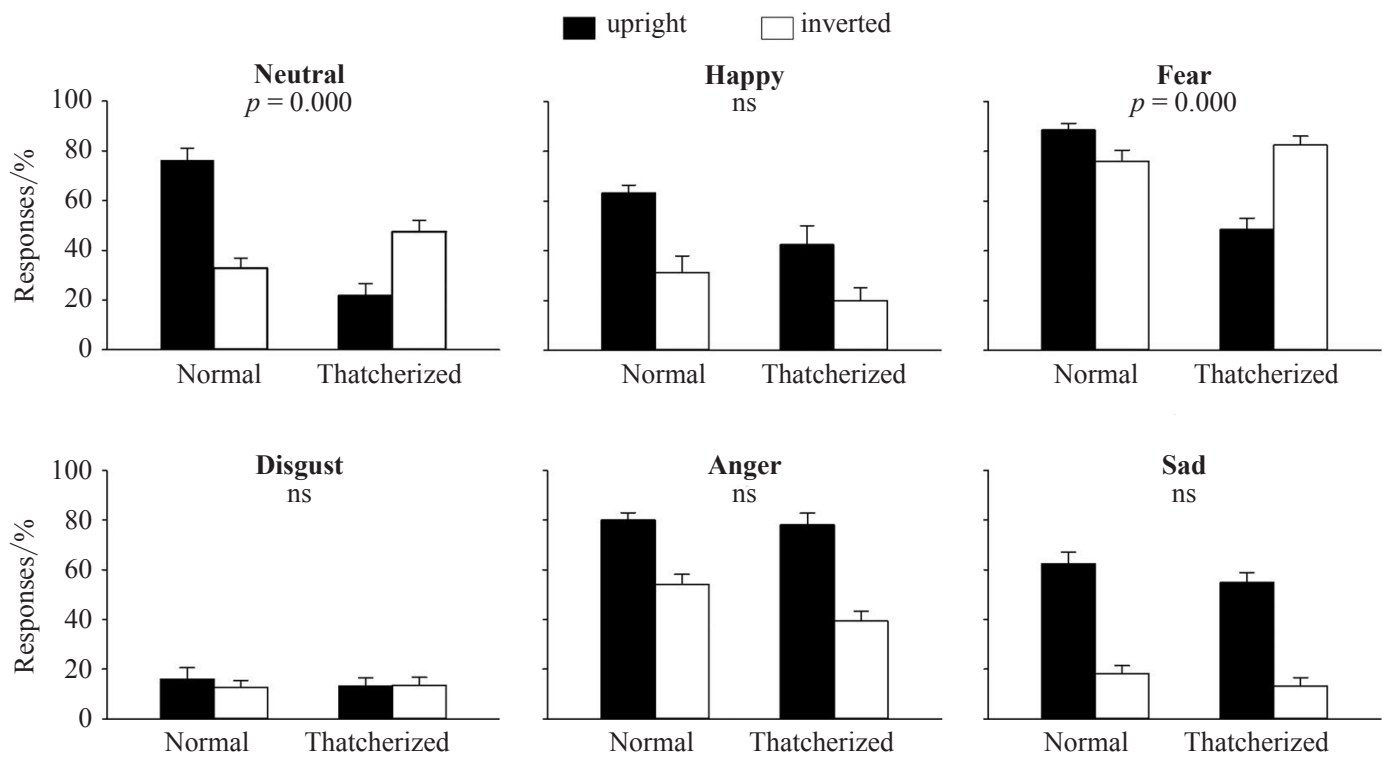

Figure 4. Recognition of facial expression in the eye region. Normal and thatcherized images were presented in an upright or inverted orientation. There was a significant interaction between the orientation of the image and whether the image was normal or thatcherized. This effect was due to lower recognition of inverted compared with upright normal faces, but higher recognition of inverted compared with upright thatcherized faces. The $p$-values for the interactions are shown for each emotional expression. Error bars represent \pm standard error across participants.

For neutral, there was a significant effect of condition $\left(F_{1,19}=36.9, p<0.001\right)$. The effect of condition was due to lower recognition of thatcherized (34.7 \pm 4.7$)$ compared with normal $(54.6 \pm 44)$ faces. There was also an effect of orientation $\left(F_{1,19}=14.9, p<0.001\right)$ and a significant interaction between condition $\times$ orientation $\left(F_{1,19}=111.9, p<0.001\right)$. This interaction is explained by lower recognition of inverted (32.8 \pm 4.1$)$ compared with upright (76.4 \pm 4.7$)$ normal images $\left(t_{19}=9.5, p<0.001\right)$, but higher recognition of inverted $(47.5 \pm 4.6)$ compared 
with upright $(21.9 \pm 4.8)$ thatcherized images $\left(t_{19}=5.1, p<0.001\right)$. Finally, the recognition of upright normal faces was significantly higher than the recognition of inverted thatcherized faces $\left(t_{19}=6.0, p<0.001\right)$.

For happy, there was a significant effect of condition $\left(F_{1,19}=40.7, p<0.001\right)$ and orientation $\left(F_{1,19}=61.6, p<0.001\right)$. The effect of condition was due to lower recognition of thatcherized $(31.3 \pm 6.4)$ compared with normal $(47.4 \pm 4.9)$ images. The effect of orientation was due to an increased recognition of upright (52.9 \pm 5.4$)$ compared with inverted $(25.7 \pm 5.9)$ images. There was no significant interaction between condition $\times$ orientation $\left(F_{1,19}=1.3, p=0.26\right)$.

For fear, there was a significant effect of condition $\left(F_{1,19}=27.1, p<0.001\right)$. The effect of condition was due to lower recognition of thatcherized (66.0 \pm 4.1$)$ compared with normal $(82.4 \pm 3.5)$ faces. There was also an effect of orientation $\left(F_{1,19}=9.5, p<0.01\right)$ and a significant interaction between condition $\times$ orientation $\left(F_{1,19}=95.8, p<0.001\right)$. This interaction is explained by the lower recognition of inverted $(76.1 \pm 4.4)$ compared with upright $(88.6 \pm 2.6)$ normal images $\left(t_{19}=3.0, p<0.01\right)$, but higher recognition for inverted $(82.5 \pm 3.7)$ compared with upright $(48.6 \pm 4.5)$ thatcherized images $\left(t_{19}=5.9, p<0.001\right)$. Finally, the recognition of upright normal faces was not significantly different from the recognition of inverted thatcherized faces $\left(t_{19}=1.7, p=0.11\right)$.

For disgust, there was no significant effect of condition $\left(F_{1,19}=0.3, p=0.60\right)$ or orientation $\left(F_{1,19}=0.6, p=0.45\right)$. There was also no significant interaction between condition $\times$ orientation $\left(F_{1,19}=0.71, p=0.41\right)$.

For anger, there was a significant effect of orientation $\left(F_{1,19}=60.2, p<0.001\right)$. The effect of orientation was due to an increased recognition of upright $(79.1 \pm 3.8)$ compared with inverted $(46.8 \pm 4.0)$ images. There was no effect of condition $\left(F_{1,19}=2.6, p=0.13\right)$ and no significant interaction between condition $\times$ orientation $\left(F_{1,19}=3.7, p=0.07\right)$.

For sad, there was a significant effect of condition $\left(F_{1,19}=5.3, p<0.05\right)$ and orientation $\left(F_{1,19}=153.3, p<0.001\right)$. The effect of condition was due to higher recognition of normal (40.4 \pm 3.8$)$ compared with thatcherized images $(34.2 \pm 3.6)$. The effect of orientation was due to higher recognition in upright $(58.8 \pm 4.2)$ compared with inverted $(15.8 \pm 3.2)$ images. However, there was no significant interaction between condition $\times$ orientation $\left(F_{1,19}=0.9, p=0.35\right)$.

To investigate the patterns of errors, a confusion matrix was generated (figure 5 ). This shows how participants responded to different emotional expressions. The pattern of incorrect responses was not obviously different for normal or thatcherized images. Rather, it appears that thatcherized and inverted images had a lower number of correct responses. For example, the correlation in correct performance between upright normal and inverted normal was $r=0.80(p<0.001)$. Similarly, the correlation in correct performance between upright normal and upright thatcherized was $r=0.80(p<0.001)$. Finally, the correlation between upright thatcherized and inverted thatcherized was $r=0.64(p<0.001)$. Together, this suggests that the effects of inversion and thatcherization reflect a lower number of correct responses rather than a different pattern of response.

3.2.2 Reaction time (eye region). A three-way ANOVA with expression (neutral, happy, fear, disgust, anger, sad), condition (normal, thatcherized), and orientation (upright, inverted) was performed on the RTs to the eye region in experiment 2 (table 2). There was a significant effect of condition $\left(F_{1,2}=31.7, p<0.05\right)$. This was due to lower RTs for normal $(1403 \pm 25 \mathrm{~ms})$ compared with thatcherized $(1510 \pm 28 \mathrm{~ms})$ faces. There was no significant effect of expression $\left(F_{5,10}=1.4, p=0.30\right)$ or orientation $\left(F_{1,2}=1.8, p=0.31\right)$. There was also no significant interaction of condition $\times$ orientation $\left(F_{1,2}=0.7, p=0.50\right)$, expression $\times$ condition $\left(F_{5,10}=1.3\right.$, $p=0.32)$, or expression $\times$ orientation $\left(F_{5,10}=1.4, p=0.31\right)$. The interaction between expression $\times$ condition $\times$ orientation was also not significant $\left(F_{5,10}=2.5, p=0.11\right)$. 


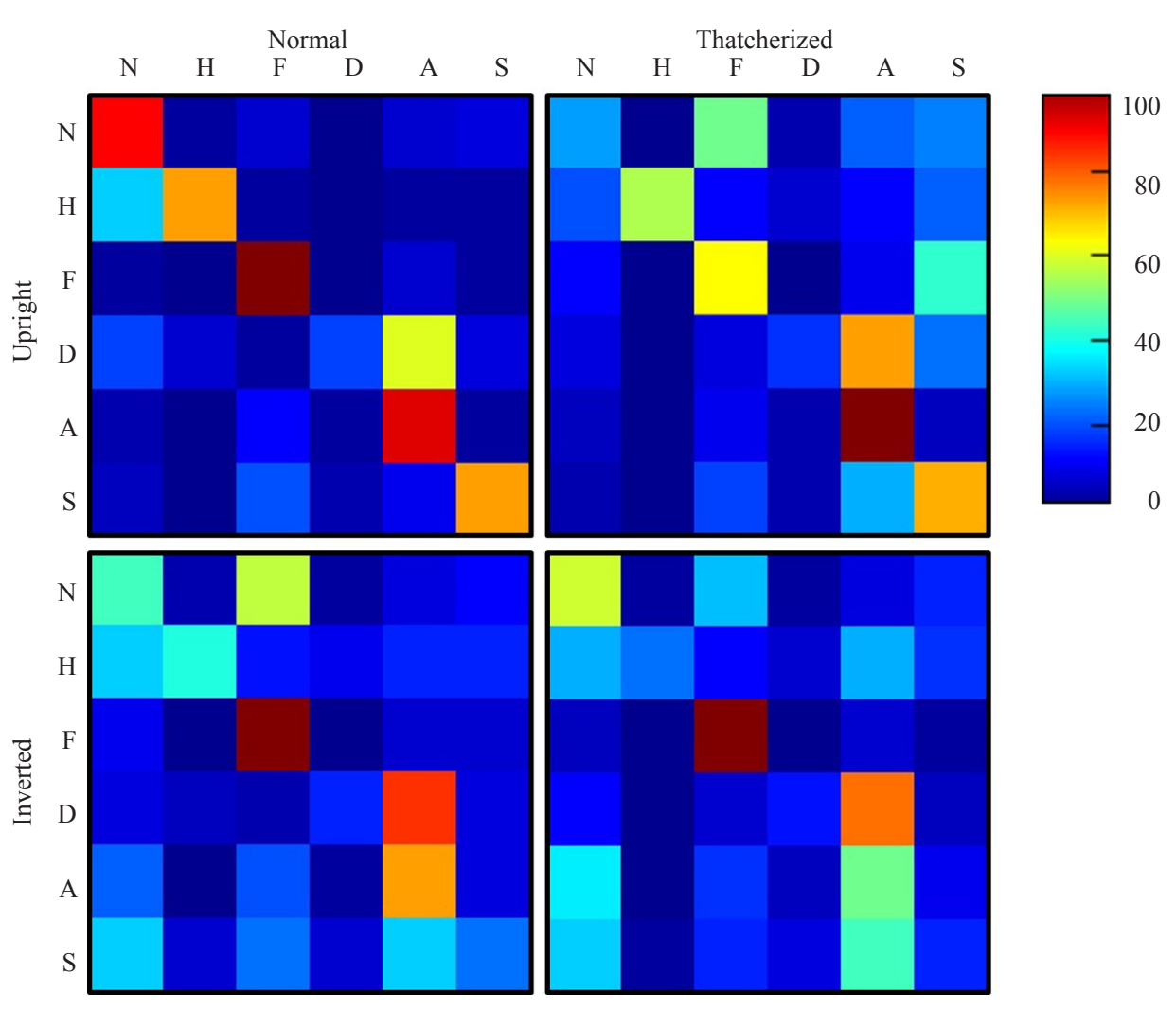

Figure 5. [In colour online.] Confusion matrices for the presented and perceived emotional expressions in the eye region. The $y$-axis represents the expression that was presented, and the $x$-axis represents the expression that was reported. Correct performance is shown along the diagonal elements, whereas errors or confusion are shown on the off-diagonal elements. The colour bar represents accuracy in $\%$. Note: $\mathrm{N}=$ neutral; $\mathrm{H}=$ happy; $\mathrm{F}=$ fear; $\mathrm{D}=$ disgust; $\mathrm{A}=$ anger; $\mathrm{S}=$ sad.

Table 2. Average reaction time values (ms) for correct responses to emotional expression when only the eye region of the face was visible in experiment 2.

\begin{tabular}{llllll}
\hline & Normal & & & \multicolumn{2}{l}{ Thatcherized } \\
\cline { 6 - 6 } \cline { 5 - 6 } & upright & inverted & & upright & inverted \\
\hline Neutral & $1166.1 \pm 64.6$ & $1462.8 \pm 100.8$ & & $1666.4 \pm 159.6$ & $1526.2 \pm 80.3$ \\
Happy & $1247.9 \pm 78.6$ & $1463.1 \pm 115.5$ & & $1335.9 \pm 66.5$ & $1488.5 \pm 106.1$ \\
Fear & $1281.7 \pm 82.4$ & $1336.2 \pm 97.6$ & & $1600.7 \pm 120.0$ & $1373.9 \pm 95.7$ \\
Disgust & $1769.9 \pm 89.8$ & $1807.8 \pm 139.9$ & & $1500.0 \pm 52.2$ & $1820.7 \pm 119.5$ \\
Anger & $1110.7 \pm 47.0$ & $1316.7 \pm 59.5$ & & $1227.2 \pm 61.6$ & $1626.1 \pm 99.1$ \\
Sad & $1331.8 \pm 52.0$ & $1546.0 \pm 111.3$ & & $1284.7 \pm 58.8$ & $1672.5 \pm 157.4$ \\
\hline
\end{tabular}

3.2.3 Recognition accuracy (mouth region). Figure 6 shows the percentage-correct recognition for each facial expression in the mouth region. To determine the effect of inversion and thatcherization on recognition of facial expressions, we performed a three-way ANOVA with expression (neutral, happy, fear, disgust, anger, sad), condition (normal, thatcherized), and orientation (upright, inverted). There were significant effects of expression $\left(F_{5,95}=86.4\right.$, $p<0.001)$, condition $\left(F_{1,19}=98.1, p<0.001\right)$, and orientation $\left(F_{1,19}=11.1, p<0.01\right)$. There was a significant interaction of condition $\times$ orientation $\left(F_{1,19}=68.4, p<0.001\right)$. This suggests that inversion has a different effect on the recognition of facial expression in normal and 

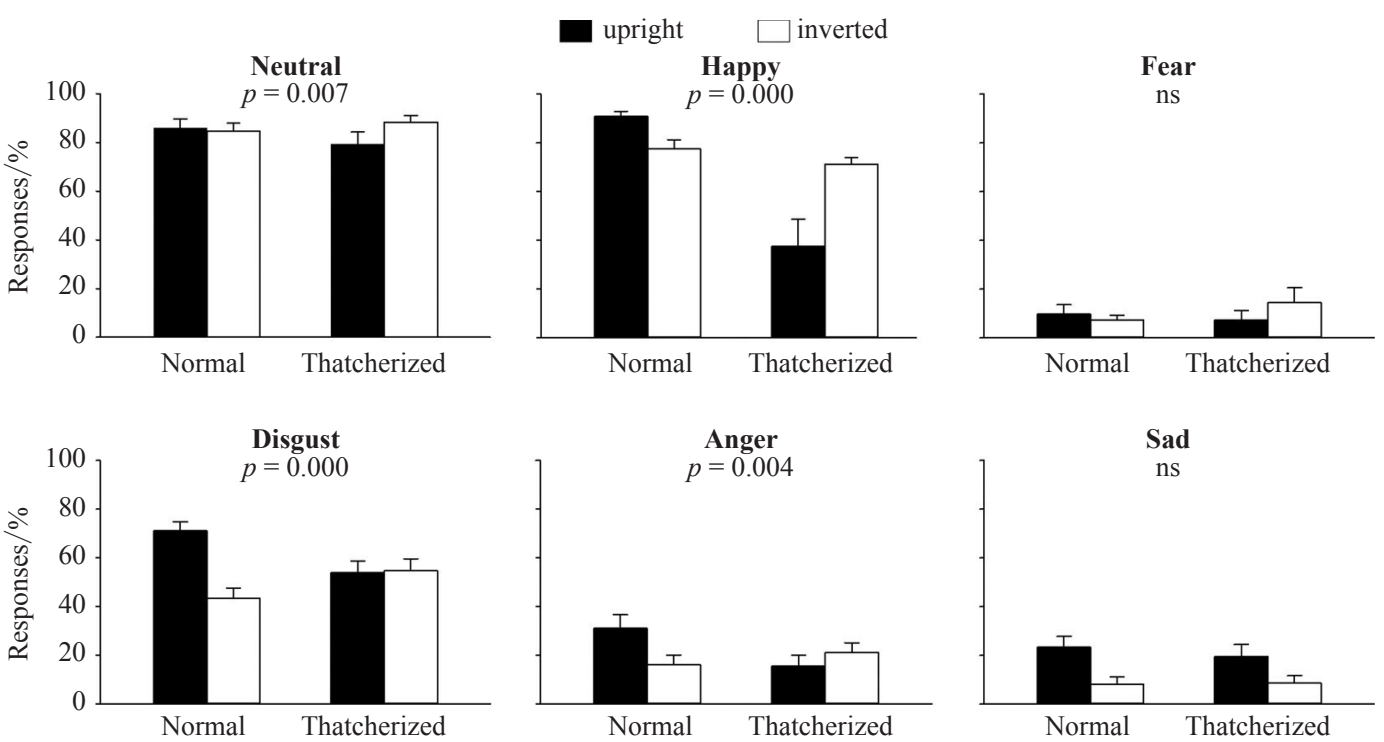

Figure 6. Recognition of facial expression in the mouth region. Normal and thatcherized images were presented in an upright or inverted orientation. There was a significant interaction between the orientation of the image and whether the image was normal or thatcherized. This effect was due to lower recognition of inverted compared with upright normal faces, but higher recognition of inverted compared with upright thatcherized faces. The $p$-values for the interactions are shown for each emotional expression. Error bars represent \pm standard error across participants.

thatcherized faces. There was also a significant interaction between expression $\times$ condition $\left(F_{5,95}=29.0, p<0.001\right)$ and expression $\times$ orientation $\left(F_{5,95}=5.1, p<0.01\right)$. To determine how the perception of different facial expressions is affected by orientation and condition, a $2 \times 2$ ANOVA was performed independently for each expression.

For neutral, there was no effect of condition $\left(F_{1,19}=0.1, p=0.33\right)$ or orientation $\left(F_{1,19}=0.6\right.$, $p=0.45)$. However, there was a significant interaction between condition $\times$ orientation $\left(F_{1,19}=9.1, p<0.01\right)$. The significant interaction is explained by no difference in recognition between inverted $(84.7 \pm 3.3)$ and upright $(86.1 \pm 3.6)$ normal faces $\left(t_{19}=0.9, p=0.38\right)$, but a higher recognition of inverted $(88.3 \pm 2.8)$ compared with upright $(79.4 \pm 4.9)$ thatcherized faces $\left(t_{19}=2.3, p<0.05\right)$. Finally, the recognition of upright normal faces was not significantly different from the recognition of inverted thatcherized faces $\left(t_{19}=-1.6, p=0.88\right)$.

For happy, there was a significant effect of condition $\left(F_{1,19}=63.1, p<0.001\right)$. The effect of condition was due to lower recognition of thatcherized (54.4 \pm 7.0$)$ compared with normal $(84.2 \pm 3.0)$ faces. There was also an effect of orientation $\left(F_{1,19}=0.4, p=0.56\right)$ and a significant interaction between condition $\times$ orientation $\left(F_{1,19}=19.9, p<0.001\right)$. This interaction was due to lower recognition of inverted $(77.5 \pm 3.8)$ compared with upright $(90.8 \pm 2.2)$ normal faces $\left(t_{19}=3.6, p<0.01\right)$, but higher recognition of inverted $(71.3 \pm 2.7)$ compared with upright $(37.5 \pm 11.2)$ thatcherized images $\left(t_{19}=2.3, p<0.05\right)$. Finally, the recognition of upright normal faces was significantly higher than the recognition of inverted thatcherized faces $\left(t_{19}=6.3, p<0.001\right)$.

For fear, there was no significant effect of condition $\left(F_{1,19}=2.5, p=0.13\right)$ or orientation $\left(F_{1,19}=1.5, p=0.24\right)$. There was also no significant interaction between condition $\times$ orientation $\left(F_{1,19}=3.7, p=0.07\right)$.

For disgust, there was no significant effect of condition $\left(F_{1,19}=3.6, p=0.07\right)$. However, there was a significant effect of orientation $\left(F_{1,19}=20.5, p<0.001\right)$ and a significant interaction between condition $\times$ orientation $\left(F_{1,19}=52.8, p<0.001\right)$. This interaction was due to lower recognition of inverted $(43.3 \pm 4.2)$ compared with upright $(71.1 \pm 3.7)$ normal 
images $\left(t_{19}=7.7, p<0.001\right)$, but no significant difference in recognition between inverted $(54.7 \pm 4.8)$ and upright $(53.9 \pm 4.8)$ thatcherized images $\left(t_{19}=1.8, p=0.08\right)$. Finally, the recognition of upright normal faces was significantly higher than the recognition of inverted thatcherized faces $\left(t_{19}=4.2, p<0.001\right)$.

For anger, there was a significant effect of condition $\left(F_{1,19}=8.7, p<0.01\right)$. The effect of condition was due to lower recognition of thatcherized (18.4 \pm 4.2$)$ compared with normal $(23.6 \pm 4.7)$ faces. There was also an effect of orientation $\left(F_{1,19}=7.7, p<0.05\right)$ and a significant interaction between condition $\times$ orientation $\left(F_{1,19}=10.4, p<0.01\right)$. This interaction was due to lower recognition of inverted (16.1 \pm 3.9$)$ compared with upright $(31.1 \pm 5.5)$ normal images $\left(t_{19}=3.5, p<0.01\right)$, but no difference in recognition of inverted $(21.1 \pm 3.9)$ compared with upright $(15.6 \pm 4.4)$ thatcherized images $\left(t_{19}=1.9, p=0.07\right)$. Finally, the recognition of upright normal faces was significantly higher than the recognition of inverted thatcherized faces $\left(t_{19}=3.4, p<0.05\right)$.

For sad, there was no significant effect of condition $\left(F_{1,19}=0.9, p=0.35\right)$, but there was a significant effect of orientation $\left(F_{1,19}=20.8, p<0.001\right)$. The effect of orientation was due to lower recognition of inverted $(8.4 \pm 3.0)$ compared with upright $(21.4 \pm 4.7)$ images. There was no significant interaction between condition $\times$ orientation $\left(F_{1,19}=1.0, p=0.32\right)$.

To investigate the patterns of errors, a confusion matrix was generated (figure 7 ). This shows how participants responded to different emotional expressions. The pattern of incorrect responses was not obviously different for normal or thatcherized images. Rather, it appears that thatcherized and inverted images had a lower number of correct responses. For example,

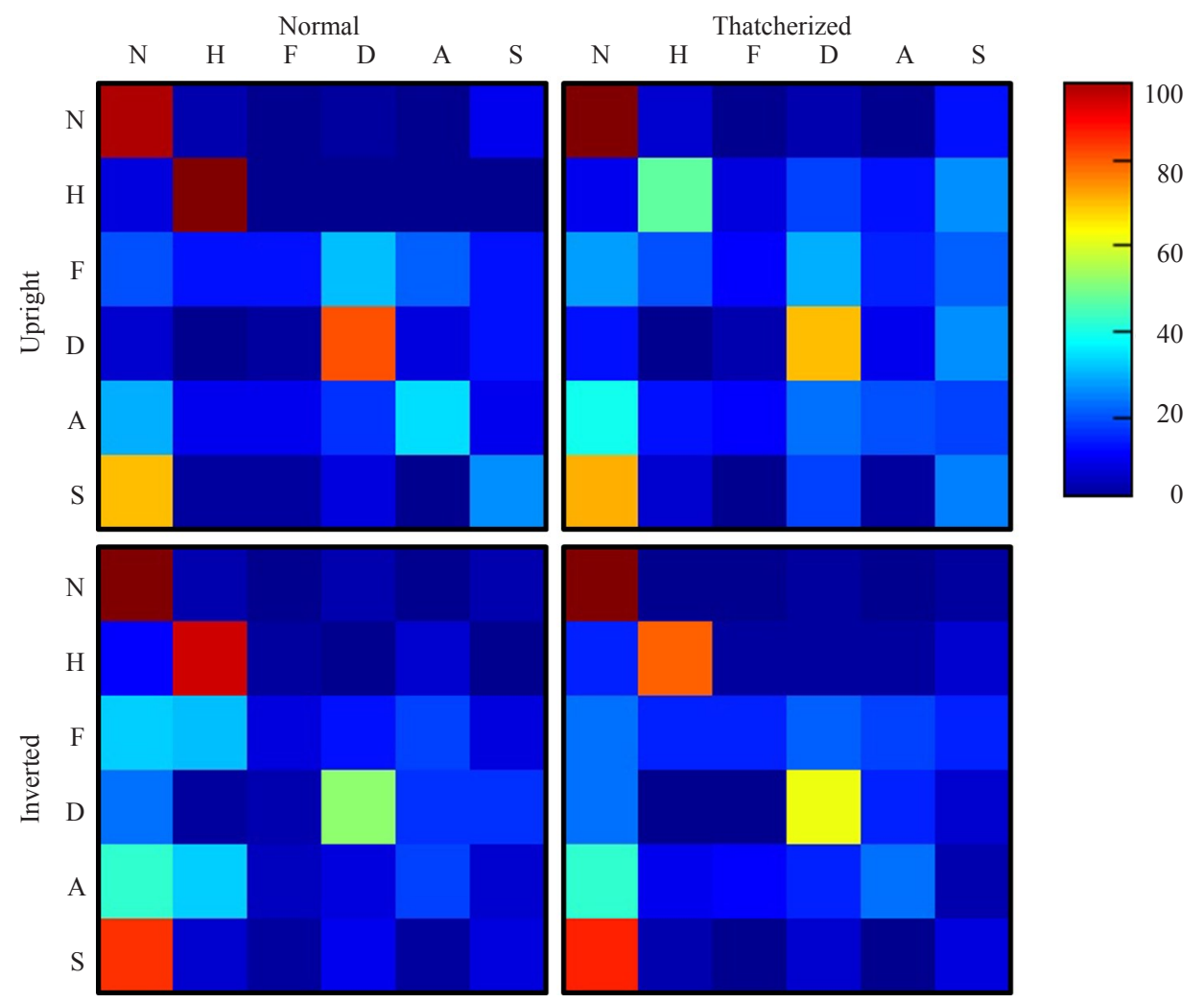

Figure 7. [In colour online.] Confusion matrices for the presented and perceived emotional expressions in the mouth region. The $y$-axis represents the expression that was presented, and the $x$-axis represents the expression that was reported. Correct performance is shown along the diagonal elements, whereas errors or confusion are shown on the off-diagonal elements. The colour bar represents accuracy in $\%$. Note: $\mathrm{N}=$ neutral; $\mathrm{H}=$ happy; $\mathrm{F}=$ fear; $\mathrm{D}=$ disgust; $\mathrm{A}=$ anger; $\mathrm{S}=$ sad. 
the correlation in correct performance between upright normal and inverted normal was $r=0.92(p<0.001)$. Similarly, the correlation in correct performance between upright normal and upright thatcherized was $r=0.89(p<0.001)$. Finally, the correlation between upright thatcherized and inverted thatcherized was $r=0.92(p<0.001)$. Together, this suggests that the effects of inversion and thatcherization reflect a lower number of correct responses rather than a different pattern of response.

3.2.4 Reaction time (mouth region). A three-way ANOVA with expression (neutral, happy, fear, disgust, anger, sad), condition (normal, thatcherized), and orientation (upright, inverted) was performed on the RT data from the mouth region in experiment 2 (table 3 ). There was a significant effect of orientation $\left(F_{1,9}=27.3, p<0.001\right)$. This was due to lower RTs for upright $(1258 \pm 24 \mathrm{~ms})$ compared with inverted $(1583 \pm 39 \mathrm{~ms})$ faces. There was an effect of expression $\left(F_{5,45}=13.8, p<0.001\right)$. This was due to differences in RTs across different expressions (neutral: $1049 \pm 37 \mathrm{~ms}$; happy: $1153 \pm 37 \mathrm{~ms}$; fear: $1731 \pm 65 \mathrm{~ms}$; disgust: $1284 \pm 41 \mathrm{~ms}$; anger: $1715 \pm 71 \mathrm{~ms}$; sad: $1591 \pm 73 \mathrm{~ms}$ ). There was not any significant effect of condition $\left(F_{1,9}=1.8, p=0.21\right)$. There was also no significant interaction of condition $\times$ orientation $\left(F_{1,9}=4.6, p=0.06\right)$, expression $\times$ condition $\left(F_{5,45}=1.3, p=0.30\right)$, or expression $\times$ orientation $\left(F_{5,45}=3.0, p=0.07\right)$. The interaction between expression $\times$ condition $\times$ orientation was also not significant $\left(F_{5,45}=2.9, p=0.11\right)$.

Table 3. Average reaction time values (ms) for correct responses to emotional expression when only the mouth region of the face was visible in experiment 2.

\begin{tabular}{llllll}
\hline & \multicolumn{2}{l}{ Normal } & & \multicolumn{2}{l}{ Thatcherized } \\
\cline { 2 - 3 } & upright & inverted & & upright & inverted \\
\hline Neutral & $922.1 \pm 52.8$ & $1084.0 \pm 71.9$ & & $1017.6 \pm 45.8$ & $1171.6 \pm 123.9$ \\
Happy & $999.2 \pm 42.2$ & $1209.4 \pm 92.3$ & & $1125.7 \pm 66.2$ & $1276.1 \pm 96.1$ \\
Fear & $1750.2 \pm 124.6$ & $1897.0 \pm 132.9$ & & $1291.4 \pm 90.4$ & $1985.4 \pm 172.1$ \\
Disgust & $1057.3 \pm 49.5$ & $1299.0 \pm 62.4$ & & $1288.1 \pm 95.9$ & $1492.7 \pm 119.8$ \\
Anger & $1513.5 \pm 102.2$ & $1977.6 \pm 190.6$ & & $1525.8 \pm 128.4$ & $1842.6 \pm 143.2$ \\
Sad & $1280.5 \pm 119.4$ & $1771.2 \pm 194.5$ & & $1320.9 \pm 70.9$ & $1989.7 \pm 202.9$ \\
\hline
\end{tabular}

\section{Discussion}

The aim of this experiment was to determine the effect of inversion on the recognition of normal and thatcherized facial expressions. Inversion of normal faces reduced the recognition of some facial expressions (disgust, anger, sad), but had no effect on the recognition of other expressions (neutral, happy, fear). In contrast, local inversion of facial features in thatcherized faces reduced the recognition of all expressions. Paradoxically, however, there was a benefit of inversion for the majority of thatcherized expressions. For some expressions (neutral, happy, fear) there was an improved recognition, whereas for other expressions (disgust, anger) there was an attenuation of the inversion effect found in normal faces. A similar pattern of results was found when only the eyes or mouth was visible. This suggests that a disruption to configural processing does not explain the Thatcher illusion.

A variety of behavioural evidence has shown that the perception of facial identity is affected by the inversion of the image (Rossion \& Boremanse, 2008; Tanaka \& Farah, 1991, 1993; Valentine, 1988; Yin, 1969). In contrast, studies of facial expression have reported only small effects of inversion, with the recognition of some emotions being completely unaffected (Birgit, Seidel, Kainz, \& Carbon, 2009; Calvo \& Nummenmaa, 2008; Fallshore \& Bartholow, 2003; McKelvie, 1995; Prkachin, 2003). Our results showed 
that inversion affected the recognition of some facial expressions (disgust, anger, sad), but it had no significant effect on the recognition of other expressions (neutral, happy, fear). The dissociation in the effect of inversion on identity and expression suggests that different representations underpin these aspects of face processing. This is consistent with a variety of evidence that suggests these facial attributes are processed along parallel processing streams (Bruce \& Young, 2012; Harris, Young, \& Andrews, 2014; Haxby, Hoffman, \& Gobbini, 2000; Young \& Bruce, 2011).

In contrast to inversion of the whole face, the local inversion of facial features in a thatcherized face had a marked effect on the recognition of all facial expressions. Given the effect of inversion on normal faces, the prediction was that there should be some reduction in the recognition of facial expressions in inverted Thatcherized faces. In contrast, we found that there was a benefit of inversion for thatcherized faces in five of the six expressions. For expressions that showed no effect of inversion in normal faces (neutral, happy, fear) there was an increased recognition of inverted compared with upright thatcherized faces. On the other hand, expressions in which there was a reduction in recognition following inversion (disgust, anger) showed no inversion effect for thatcherized faces.

So, what explains the different effect of inversion on normal and thatcherized faces. One possible explanation is that the orientation of the eyes and mouth in an inverted, thatcherized face is in the correct orientation. So, if the recognition of facial expression is based solely on the orientation of the expressive features of the face, then the features may be more recognizable in the typical orientation. However, this explanation would predict that the recognition of expression in inverted thatcherized images should be equivalent to upright normal faces. The results show that recognition of facial expression for inverted, thatcherized faces is typically lower than for upright, normal faces.

Another possible explanation for the improved recognition of facial expression in inverted thatcherized faces could be the way that facial expression is encoded. A variety of evidence suggests that the perception of facial expression can be based on either a continuous (Russell \& Bullock, 1985; Woodworth \& Schlosberg, 1954) or a categorical (Darwin, 1998; Ekman, 1972) representation. In a recent study we provided a neural explanation for these findings by showing that a face-selective region in the posterior superior temporal sulcus (pSTS) had a continuous representation of facial expression, whereas the face-selective region of the amygdala had a more categorical representation of facial expression (Harris, Young, \& Andrews, 2012). It is possible, therefore, that these representations may be differentially affected by inversion. We provided partial support for this possibility in a recent study in which we showed that pSTS was sensitive to the orientation of thatcherized faces (Psalta, Young, Thompson, \& Andrews, 2014). In contrast, the categorical representation of expression in regions such as the amygdala may have a coarser scale that is less sensitive to orientation (Vuilleumier, Armony, Driver, \& Dolan, 2003). For example, an increase in contrast in the mouth region could indicate happiness, whereas an increase in contrast in the eye region could indicate fear. Differences in the effect of inversion on each expression may reflect differential sensitivity of the key visual information that is diagnostic of these different facial expressions. From this perspective, the reduced recognition of facial expression in upright thatcherized faces could result from interference between different neural representations of facial expression. When the faces are inverted, the orientation-sensitive representation that gives rise to the grotesque expression is attenuated but the less orientation-sensitive categorization representation of facial expression continues to be processed.

The effect of inversion on the perception of facial expression seen in the Thatcher illusion is widely attributed to the absence of configural processing in inverted faces (Bartlett \& Searcy, 1993; Boutsen \& Humphreys, 2003; Boutsen, Humphreys, Praamstra, \& Warbrick, 2006; Leder, Candrian, Huber, \& Bruce, 2001; Lewis \& Johnston, 1997; Rhodes, Brake, \& Atkinson, 1993). 
To address whether the effects that we have observed could also be explained by the configural properties of the face, we repeated the experiment with only the mouth region or the eye region visible. If the pattern of results can be explained by configural processing, we would expect that they would be abolished when only the isolated features are visible and there is no configural information. However, we found a similar interaction between the effect of inversion on normal and thatcherized images. When only the eye region was shown, there was a significant interaction between the effect of thatcherization and the effect of inversion for two expressions (neutral and fear). This interaction occurred because inversion of neutral or fear faces resulted in a reduction in the recognition of normal faces, but an increased recognition of thatcherized faces. When only the mouth region was shown, there was a significant interaction between the effect of thatcherization and inversion on three expressions (neutral, happy, disgust). Inversion resulted in a reduction in recognition in normal happy faces, but an increased recognition in thatcherized happy faces. Inversion had no effect on normal neutral faces, but increased recognition of thatcherized neutral faces. Finally, inversion had a significant reduction on the recognition of normal disgust faces, but had no effect on the recognition of thatcherized disgust faces. The differences in which expressions showed an interaction between orientation and thatcherization for the eye and mouth region reflect the relative importance of these regions for different expressions. Indeed, overall recognition of facial expressions also varied as a function of facial feature. For example, the recognition of fear was more accurately recognized from the eye region, whereas disgust and happy were more easily recognized from the mouth region.

Our findings are consistent with recent studies showing a lack of evidence for configural processing of upright thatcherized faces, as defined by RT-based (Donnelly, Cornes, \& Menneer, 2012) and accuracy-based (Mestry, Menneer, Wenger, \& Donnelly, 2012) measures. In experiment 2 the only cue to the orientation of the face was the jaw line for the mouth region and the eyebrows or the bridge of the nose for the eye region. Nevertheless, it appears that these cues are sufficient to signal the critical orientation cues that influence our perception of the facial features. The presence of interactions between orientation and thatcherization when only the eye or mouth regions were shown suggests that inversion is disrupting the local coding of the expressive features of the face. The findings suggest that the perception of facial features can be influenced by the context in which the face is perceived. This fits with a recent study that demonstrated how the global properties (including orientation) of natural images (including faces) can influence feature detectors (Neri, 2011, 2014).

In conclusion, we show that the perception of facial effect of inversion on normal faces varied for different expressions. There was a significant effect of inversion on some expressions, but little or no effect on the recognition of other expressions. In contrast to inversion, thatcherization of images significantly reduced recognition across all emotional expressions. Interestingly, however, we found that inverting thatcherized images actually improved recognition of some facial expressions. We suggest that this paradoxical improvement in face perception with inversion may provide insights into the way that different visual information is represented for the processing of different aspects of face perception.

Acknowledgment. This work was supported by a grant from the Wellcome Trust (WT087720MA).

\section{References}

Bartlett, J. C., \& Searcy, J. (1993). Inversion and configuration of faces. Cognitive Psychology, 25, 281-316.

Birgit, D., Seidel, E. M., Kainz, E., \& Carbon, C. C. (2009). Recognition of emotional expression is affected by inversion and presentation time. Perception, 38, 1849-1862.

Boutsen, L., \& Humphreys, G. W. (2003). The effect of inversion on the encoding of normal and "Thatcherized" faces. Quarterly Journal of Experimental Psychology Section A: Human Experimental Psychology, 56, 955-975. 
Boutsen, L., Humphreys, G. W., Praamstra, P., \& Warbrick, T. (2006). Comparing neural correlates of configural processing in faces and objects: An ERP study of the Thatcher illusion. NeuroImage, 32, 352-367.

Bruce, V., \& Young, A. (2012). Face perception. Hove, East Sussex: Psychology Press.

Calvo, M. G., \& Nummenmaa, L. (2008). Detection of emotional faces: Salient physical features guide effective visual search. Journal of Experimental Psychology: General, 137, 471-494.

Carey, S., \& Diamond, R. (1977). From piecemeal to configurational representation of faces. Science, $195,312-314$.

Darwin, C. (1998). The expression of the emotions in man and animals. P. Ekman (Ed.). London: HarperCollins.

Diamond, R., \& Carey, S. (1986). Why faces are and are not special: An effect of expertise. Journal of Experimental Psychology: General, 115, 107-117.

Donnelly, N., Cornes, K., \& Menneer, T. (2012). An examination of the processing capacity of features in the Thatcher illusion. Attention, Perception, \& Psychophysics, 74, 1475-1487.

Ekman, P. (1972). Universals and cultural differences in facial expressions of emotion. In J. Cole (Ed.), Nebraska Symposium on Motivation (pp. 207-282). Lincoln, NE: University of Nebraska Press.

Fallshore, M., \& Bartholow, J. (2003). Recognition of emotion from inverted schematic drawings of faces. Perceptual \& Motor Skills, 96, 236-244.

Goren, D., \& Wilson, H. R. (2006). Quantifying facial expression recognition across viewing conditions. Vision Research, 24, 1253-1262.

Harris, R., Young, A., \& Andrews, T. (2012). Morphing between expressions dissociates continuous from categorical representations of facial expression in the human brain. Proceedings of the National Academy of Sciences, 109, 21164-21169.

Harris, R. J., Young, A. W., \& Andrews, T. J. (2014). Brain regions involved in processing facial identity and expression are differentially selective for surface and edge information. NeuroImage 97, 217-223.

Haxby, J. V., Hoffman, E. A., \& Gobbini, M. I. (2000). The distributed human neural system for face perception. Trends in Cognitive Sciences, 4, 223-233.

Leder, H., Candrian, G., Huber, O., \& Bruce, V. (2001). Configural features in the context of upright and inverted faces. Perception, 30, 73-83.

Lewis, M. B., \& Johnston, R. A. (1997). The Thatcher illusion as a test of configural disruption. Perception, 26, 225-227.

McKelvie, S. J. (1995). Emotional expression in upside-down faces: Evidence for configurational and componential processing. British Journal of Social Psychology, 34, 325-334.

Mestry, N., Menneer, T., Wenger, M. J., \& Donnelly, N. (2012). Identifying sources of configurality in three face processing tasks. Frontiers in Psychology, 3, 456.

Neri, P. (2011). Global properties of natural scenes shape local properties of human edge detectors. Frontiers in Psychology, 2, 1-20.

Neri, P. (2014). Semantic control of feature extraction from natural scenes. Journal of Neuroscience, 34, 2374-2388.

Prkachin, G. (2003). The effects of orientation on detection and identification of facial expressions of emotions. British Journal of Psychology, 94, 45-62.

Psalta L., Young A. W., Thompson P., \& Andrews T. J. (2014). The Thatcher illusion reveals orientation dependence in brain regions involved in processing facial expression. Psychological Science, 25, $128-136$.

Rhodes, G., Brake, S., \& Atkinson, P. (1993). What's lost in inverted faces? Cognition, 47, 25-57.

Rossion, B., \& Boremanse, A. (2008). Nonlinear relationship between holistic processing of individual faces and picture-plane rotation: Evidence from the face composite illusion. Journal of Vision, 8(3):4, 1-13.

Russell, J. A., \& Bullock, M. (1985). Multidimentional scaling of emotional facial expressions: Similarities from pre-schoolers to adults. Journal of Personality and Social Psychology, 48, 1290-1298.

Tanaka, J. W., \& Farah, M. (1993). Parts and wholes in face recognition. Quarterly Journal of Experimental Psychology Section A: Human Experimental Psychology, 46, 225-245. 
Tanaka, J. W., \& Farah, M. J. (1991). Second-order relational properties and the inversion effect: Testing a theory of face perception. Perception \& Psychophysics, 50, 367-372.

Thompson, P. (1980). Margaret Thatcher: A new illusion. Perception, 9, 483-484.

Valentine, T. (1988). Upside-down faces - a review of the effect of inversion upon face recognition. British Journal of Psychology, 79, 471-491.

Vuilleumier, P., Armony, J. L., Driver, J., \& Dolan, R. J. (2003). Distinct spatial frequency sensitivities for processing faces and emotional expressions. Nature Neuroscience, 6, 624-631.

Woodworth, R. S., \& Schlosberg, H. (1954). Experimental Psychology. New York: Henry Holt.

Yin, R. K. (1969). Looking at upside-down faces. Journal of Experimental Psychology, 81, 141-145.

Young, A., \& Bruce, V. (2011). Understanding person perception. British Journal of Psychology, 102, 959-974.

Young, A. W., Perrett, D. I., Calder, A. J., Sprengelmeyer, R., \& Ekman, P. (2002). Facial Expressions of Emotion-Stimuli and Tests (FEEST). Bury St Edmunds, Suffolk: Thames Valley Test Company. 